\title{
Efficiency of Schemes of Active Broadband Damping of Vibration Forces Transmitted by Vibration Insulation to the Foundation
}

\author{
Aleksey Vladimirovich Kirjukhin ${ }^{1}$, Oleg Osherevich Milman ${ }^{2}$, Anton Viktorovich Ptakhin ${ }^{3}$, Vasily \\ Dmitrievitch Kuprjashov' \\ ${ }^{1}$ Kaluga State Tsiolkovsky University, Kaluga, Russia; SPC Turbocon, Kaluga, Russia \\ ${ }^{2}$ Kaluga State Tsiolkovsky University, Kaluga, Russia; SPC Turbocon, Kaluga, Russia \\ ${ }^{3}$ Kaluga State Tsiolkovsky University, Kaluga, Russia; SPC Turbocon, Kaluga, Russia
}

${ }^{4}$ SPC Turbocon, Kaluga, Russia

\begin{abstract}
Five possible schemes of the active vibration protection system (AVS) designed to damp vibration forces transmitted to the foundation through the vibration isolation of the installation in a wide frequency range have been considered. The scheme influence on the effectiveness and physical parameters of the original system, such as the rigidity of vibration isolation, mass, natural (self-resonant) vibration frequency has been researched. For clarity the analysis was performed for a single-mass mechanical system. The calculation has defined the influence of the AVS scheme on the amplitude-frequency characteristic of the force transmitted to the foundation. The physical causes of this effect have been analyzed, and dependences for the AVS efficiency have been obtained. Proposals for choosing the most effective AVS schemes have been made.
\end{abstract}

Key words: active vibration protection system (AVS), vibrational force, vibration isolator, force sensor, transfer function, gain, feedback.

\section{INTRODUCTION}

In industry, transport and energy production there are requirements to be vibration of the structures attached to vibration active mechanisms. As a rule, vibration damping over a wide frequency range from several to hundreds of hertz is necessary. The possibility to reduce these vibrations by reducing the rigidity of vibration isolation is limited by the allowable amount of displacement from external forces. Active vibration protection systems (AVS) allow reducing these vibrations additionally at a constant static rigidity of the vibration isolation [1 -3]. Scientific papers reviewed different schemes of AVS, intended to reduce the vibration of both the mechanisms and vibrational forces transferred to the foundation through a vibration isolation. A review of such papers is given in [1-4]. Schemes with feedback and compensation systems [1,2] have been researched, as well as the effect of various factors such as mass and compliance of the foundation, the dynamic properties of the active vibration damper, the damping effect etc., which makes the comparison quite problematic. A clear comparative analysis of AVS schemes is needed in terms of their effectiveness in a wide frequency range.

\section{PROPOSED METHODOLOGY}

The paper has researched the calculated efficiency of five possible broadband AVS schemes. In order to maximize the illustrativeness, a symmetric single - mass system has been considered (Figures 1-5). It is assumed that the vibrational rigidity of the foundation is much greater than the rigidity of the vibration isolator, which is assumed to be independent of frequency. The schemes differ in the application point of the compensating active force (to the vibration-isolated mass or the foundation) and in the way the control signal is set (by the vibrational force transferred to the foundation or by the mass vibration). Comparison is made by the magnitude of the reduction of the vibrational force transferred to the foundation through a vibration isolator. Due to such simplification, it is possible to determine the ultimate efficiency of the schemes and clearly show their difference and effect on the physical parameters of the initial mechanical system.

\section{RESULT ANALYSIS}

3.1 Compensating Force Is Applied to The Mass, Control Signal Is Taken from A Force Sensor Under the Vibration Isolator, Figure 1 A. (Scheme 1)

Mass $M$ is mounted on the vibration isolators with an accumulated rigidity $S$. The vibration isolators are fastened to the foundation with vibration rigidity $C f$ through the vibrational force sensors with the accumulated rigidity $C d$, which exceeds considerably the vibration isolation rigidity $(C d>C, C f>C)$. The mass $M$ under the action of a dynamic force $Q$ vibrates, acting through the vibration isolators on the force sensors and the foundation with force $Q f$. Accumulated signal from the force sensors is controlled with a measuring unit (e.g., a spectra analyzer) and fed to the AVS control unit with the transfer function $H$ and the gain $k$. AVS forms the accumulated active power $Q a$ acting on the mass $M$. The transfer function $H$ describes the entire control channel from the force sensors to the actuating element that implements the active compensating force $Q a$. The equations of motion, taking into account the fact that $C d>C$ 


$$
\begin{array}{r}
-M * \frac{d^{2} X}{d t^{2}}-C * X+Q-Q a=0 \\
Q a=-C * X * k * H
\end{array}
$$

The harmonic force $Q=Q \operatorname{osin}(\omega t)$ acts on the mass with a circular frequency $\omega=2 \pi f$, where $f$ is the vibration frequency, Hz. The expressions for the amplitudes of displacement $X$ o of mass $M$ and the vibrational force $Q f o$ acting on the force sensors and on the foundation will take the form

$$
X o=-\frac{Q o}{M *\left[\omega^{2}-\frac{(1-k * H) * C}{M}\right]}
$$
system $\quad p=\sqrt{\frac{C}{M}}$

$$
Q f o=C * X o=-\frac{C}{M *\left[\omega^{2}-\frac{(1-k * H) * C}{M}\right]} * Q
$$

From the formulas (1) and (2) it follows that the effect of the AVS scheme $\mathrm{ABC}$ on the vibration amplitude $X o$ of the mass $M$ is equivalent to the decrease or increase amortization rigidity $C$, depending on the sign of the gain $k$. This changes the resonant frequency of the

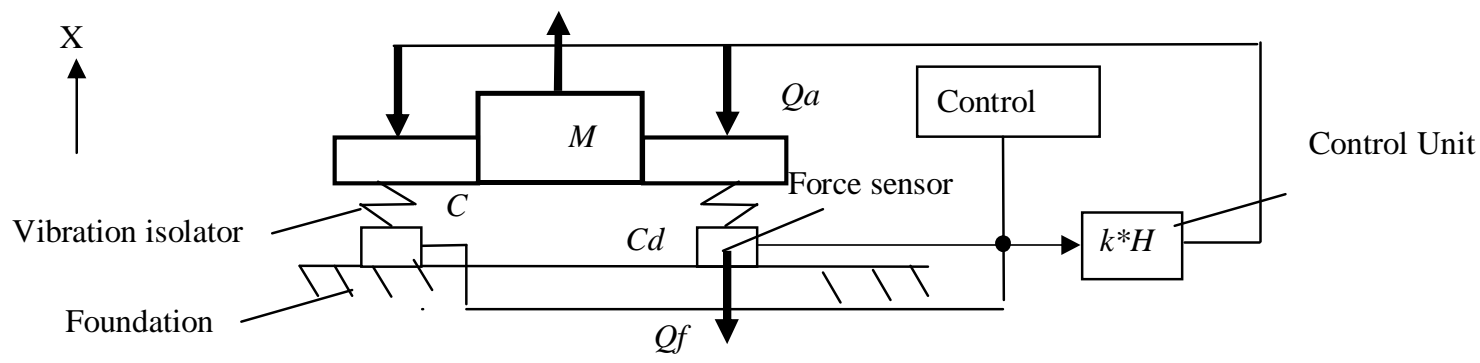

a)

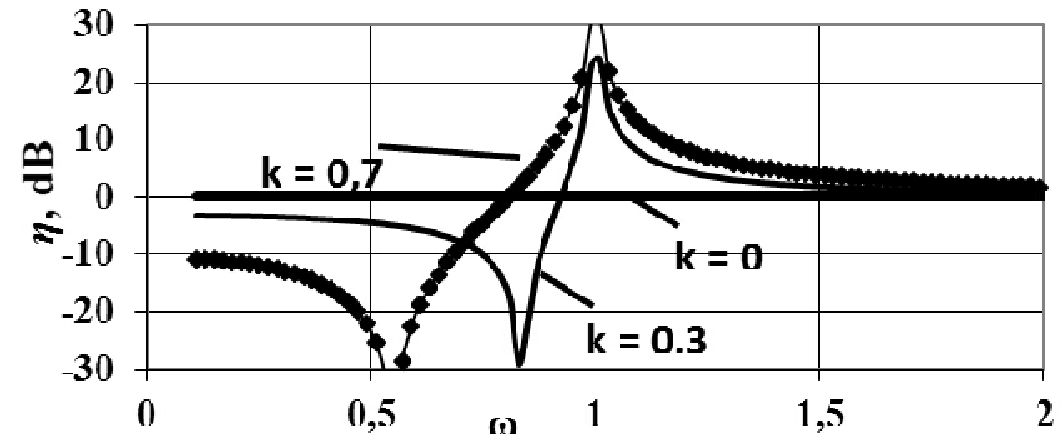

b)

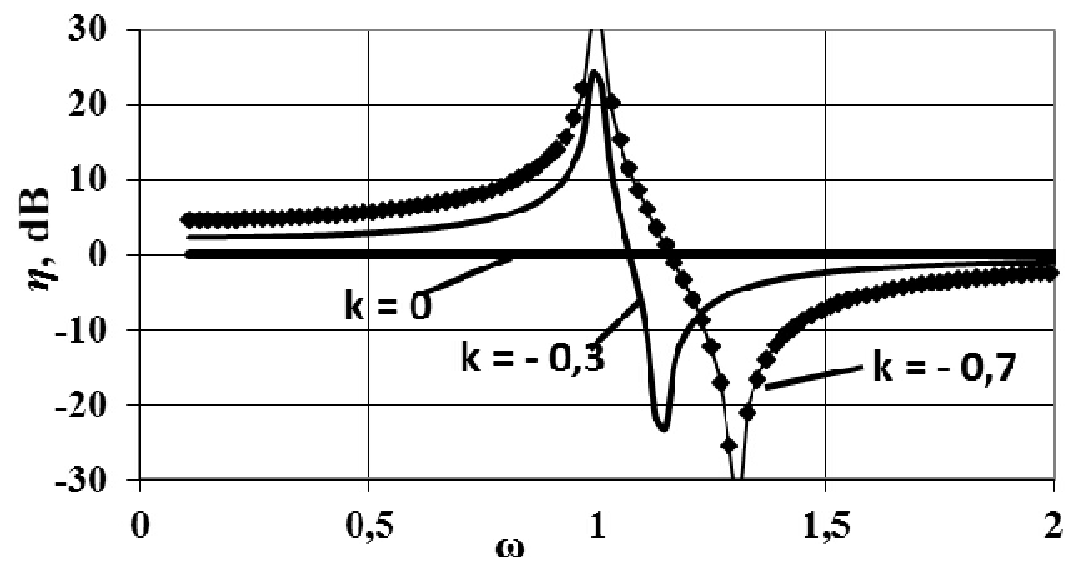

c)

Figure 1: AVS scheme $1 \mathrm{a}$ ) and its efficiency $\eta$ dependences from the non-dimensional frequency $\bar{\omega}=\frac{\omega}{p}$ at $\mathrm{k}>0$ b) and at $\mathrm{k}<0 \mathrm{c})$. 
Let the AVS efficiency is $\eta=20 * \lg \left(\frac{|Q f o(k=0)|}{|Q f o(k \neq 0)|}\right)$. At positive values $\eta$ AVS reduces the force acting on the foundation. For scheme 1 at figure 1 a

$$
\eta=20 * \lg \left(\frac{\left|\omega^{2}-p^{2}+k * H^{*} p^{2}\right|}{\left|\omega^{2}-p^{2}\right|}\right)
$$

Figures $1 b$ and $1 \mathrm{c}$ show the AVS (Scheme 1) efficiency dependences from the non-dimensional frequency $\bar{\omega}=\frac{\omega}{p}$ at $H=1$ and different gains $k$.

At $k>0$ the AVS efficiency is negative at frequencies up to the resonance $\omega<p$ due to the shifting of the new resonance frequency into the low frequency region. Maximum $\eta$ is reached at the initial resonance frequency $\omega=p$. With the frequency increase at $\omega \rightarrow \infty$ the efficiency $\eta$ tends to zero. At $k<0$ on the contrary, the resonance frequency increases, $\eta$ is positive up to the resonance and is negative in the region above the resonance.

3.2 Compensating Force Is Applied to The Mass, Control Signal Is Taken From The Acceleration Sensors (Accelerometers) On The Mass, Figure 2 A (Scheme 2)

The accumulated signal from the accelerometers mounted on the mass is fed into the AVS. Then

$$
Q a=-X * \omega^{2} * k * H
$$

The expression for amplitude of the force $Q f$ o, acting on the foundation and efficiency $\eta$ will take the form:

$$
\begin{gathered}
\text { qfo }=C * X=-\frac{C}{(M+k * H) * \omega^{2}-C} * 0 \circ \\
\eta=20 * \lg \left(\frac{\left|\omega^{2}-p^{2}+\frac{k^{*} H}{M} * \omega^{2}\right|}{\left|\omega^{2}-p^{2}\right|} \mid\right.
\end{gathered}
$$

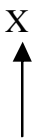

Q

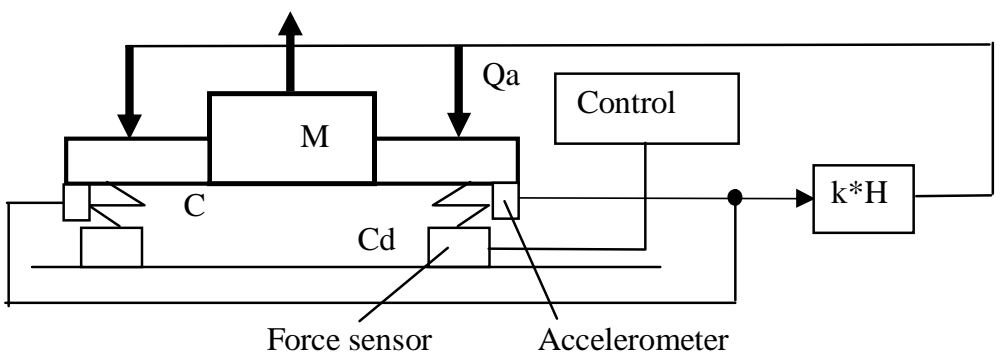

a)

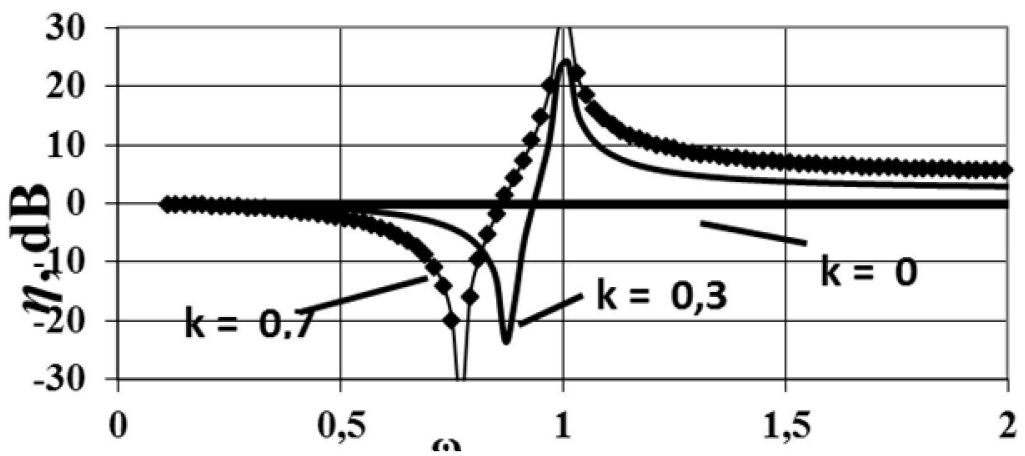

b)

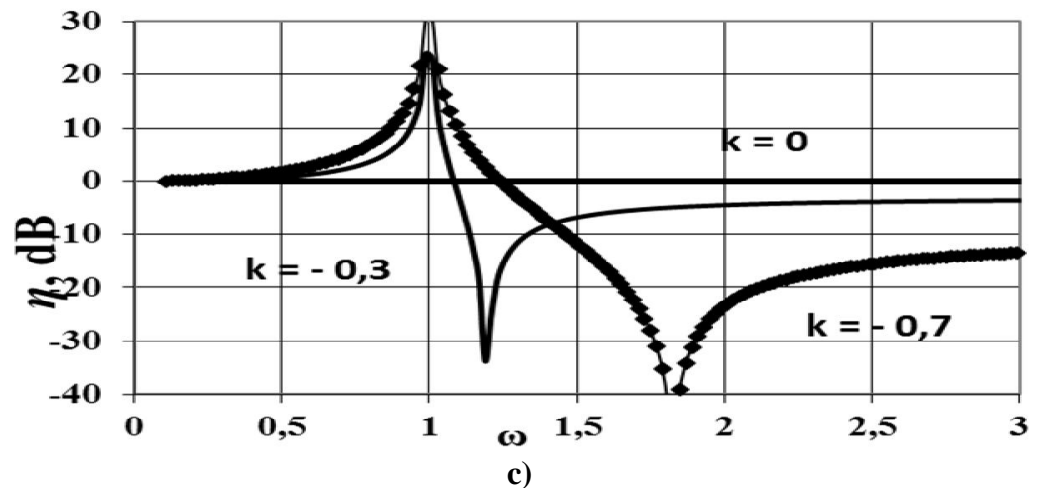

Figure 2: AVS scheme 2 a) and its efficiency $\eta$ dependences from the non-dimensional frequency $\frac{\bar{\omega}}{p}$ at $\left.\mathrm{k}>0 \mathrm{~b}\right)$ and at $\mathrm{k}<0$ c). 
For scheme № 2 the AVS effect is proportional to the mass $M$ increase or decrease depending on $k$, as a result the resonance frequency of the system changes. In this case $k$ has mass dimension. Figures $2 \mathrm{~b}$ and $2 \mathrm{c}$ show the efficiency dependence on the non-dimensional frequency

$\bar{\omega}=\frac{\omega}{p}$ and non-dimensional coefficient $\bar{k}=\frac{k}{M}$ at $H=1$ and different gains $\bar{k}$.

At $k>0$ the efficiency is negative up to the resonance at $\omega<p$ due to the shifting of the resonance frequency into the low-frequency region and is maximum at the resonance frequency $\omega=p$. With the frequency increase $\omega \rightarrow \infty$ the efficiency tends to the value $\eta=20 * \lg \left(1+\frac{k * H}{M}\right)$

At $k<0$ on the contrary, resonance frequency increases, the efficiency is positive at frequencies up to the resonance and negative above the resonance.

\subsection{Compensating Force Is Applied to The Force} Sensors, The Control Signal Is Taken from The Force

\section{Sensors Under the Vibration Isolator, Figure 3.} (Scheme № 3)

The harmonic vibration $X$ of the mass $M$ with the amplitude $X o$ is specified. Active forces $Q a$ act directly on the force sensors and through them on the foundation reducing the initial vibrational effect. The force at the force sensors is $Q d=C^{*} X-Q a$, taking into consideration that $\mathrm{Cd} \gg \mathrm{C}$. Then

$$
\begin{aligned}
& Q a=k^{*} H * Q d=k * H *(C * X-Q a) \\
& Q d=\left(1-\frac{k^{*} H}{1+k^{*} H}\right) * C * X=\frac{C}{1+k^{*} H} * X \\
& \eta=20 * \lg \left(1+k^{*} H\right)
\end{aligned}
$$

This AVS scheme at $k>0$ reduces the force acting on the foundation without effecting the dynamic characteristics of the mechanical system. The AVS efficiency does not depend explicitly on the frequency (the frequency dependence only through the transference function $H$ ), linearly depends on gain $k$ and can reach great values.

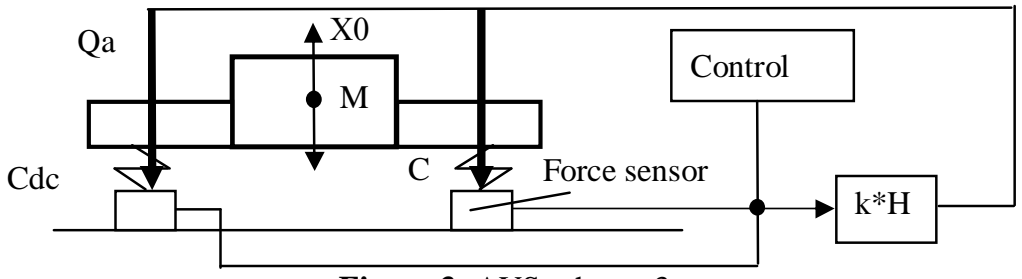

Figure 3: AVS scheme 3

3.4 Compensating Force Is Applied to The Force Sensors And The Mass, The Control Signal Is Taken From The Force Sensors Under The Vibration Isolator, Figure 4. (Scheme №4.)

The active force $Q a$ acts simultaneously both on the mass $\mathrm{M}$ and the force sensors. Such a scheme is implemented if the stator of the AVS execution unit (vibrator) is mounted on the vibro-isolated object, and the moving anchor is attached to the force sensor. In this case it is possible to create considerable dynamic strain at low frequencies due to large mechanism mass.

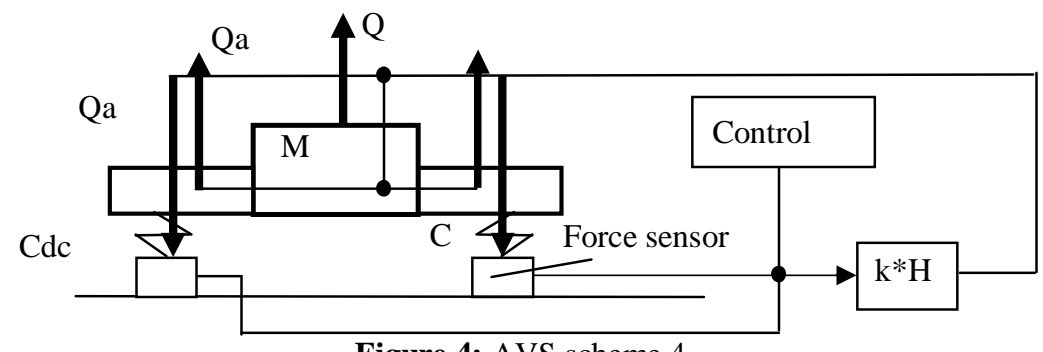

Figure 4: AVS scheme 4

Similar to sections $1-3$ the expressions for amplitudes $X o$ of mass $M$ transfer, the force $Q f$, acting on the force sensors and the foundation and the efficiency $\eta$ are obtained

$$
X o=-\frac{Q o}{M * \omega^{2}-\frac{C}{1+k * H}}
$$

$$
\begin{array}{r}
Q f o=Q d o=-\frac{\frac{C}{1+k^{*} H}}{M * \omega^{2}-\frac{C}{1+k * H}} * Q o \\
\eta=20 * \lg \left(\frac{\left|\omega^{2}-p^{2}+k^{*} H^{*} \omega^{2}\right|}{\left|\omega^{2}-p^{2}\right|}\right)
\end{array}
$$

The obtained formula (6) for the efficiency $\eta$ coincides with the similar formula (4) of the scheme №2 in the 
section 2. It follows that the dependencies of the AVS

efficiency on the non-dimensional frequency $\bar{\omega}=\frac{\omega}{p}$ at $H=1$ and different gains $k$ coincide with the dependencies at figures $2 \mathrm{~b}$ and $2 \mathrm{c}$. At $k>0$ AVS efficiency is negative in the region $\omega<p$ due to the shifting of the resonance frequency into the low frequency region and is maximum at the resonance frequency $\omega=p$ and with the frequency increase $\omega \rightarrow \infty$ tends to the value $\eta=20 * \lg (1+k * H)$. At $k<0$ on the contrary, resonance frequency increases, the efficiency is positive at $\omega<p$ and is negative in the region above the resonance. The effect of such AVS is equal to the rigidity $\mathrm{C}$ value change to the lesser or greater value depending on the sign of the coefficient $k$, as a result the mechanical system resonant frequency changes as well as the force acting on the foundation.

\subsection{The Compensating Force Is Applied to Additional Force Sensors Under the Intermediate Foundation, The Control Signal Is Taken from The Force Sensors Under the Vibration Isolator, Figure 5. (Scheme № 5)}

There are two groups of force sensors in the scheme. Under the shock absorbers with rigidity $\mathrm{C}$, the first group of force sensors (control or reference) with rigidity $C d 1>C$ is installed on the intermediate foundation (in the general case, it may be absent). A second group of force sensors (control) with rigidity $C d 2>C$ is installed on the main foundation under the first group of sensors. $X m$ is the vibration of the mechanism mass $M$. The accumulated signal from the second group of force sensors is fed to the control unit, and from the first group to the AVS with the transfer function $H$ and gain $k$, which acts on the intermediate foundation and the second group of force sensors with active force $Q a$.

In the previous schemes, the control signals from the force sensors change under the action of the active forces $Q a$, decreasing with the increasing gain $k$. These schemes are with inverse relationship, which may adversely effect the AVS resistance. In this scheme, due to the fact that $C d 2>>C$, the control signal from the first group of sensors is practically independent of the active force $Q a$, effecting the second group of force sensors and the primary foundation. It can be considered a reference signal. Such a scheme with direct relationship may provide greater resistance of AVS.

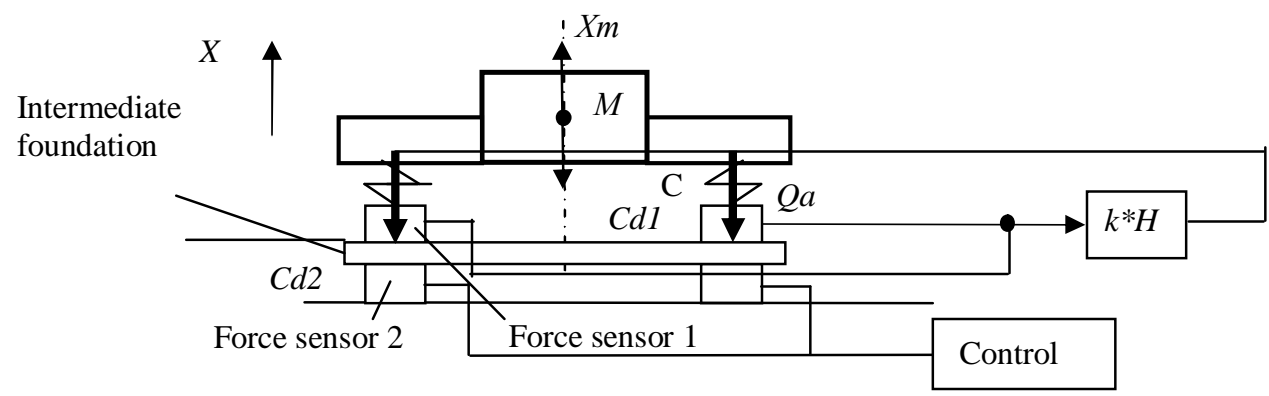

Figure 5: AVS scheme 5

Taking into consideration that $C d 1>>C$ and $C d 2>>C$ then the forces effecting the first and second group of sensors $Q 1$ and $Q 2$, can be written as $Q 1=C * X m$, $Q 2=C^{*} X m-Q a \quad$. The force $Q f$ effecting the foundation and the efficiency $\eta$ are written as

$$
\begin{array}{r}
Q f=Q 2=(1-k * H) * C * X m \\
\eta=20 * \lg \left(\left|\frac{1}{1-k^{*} H}\right|\right)
\end{array}
$$

The use of AVS according to this scheme at $0<k^{*} H<1$ reduces the dynamic force acting on the foundation without effecting the dynamic characteristics of the mechanical system. The efficiency of the AVS does not depend explicitly on the frequency (frequency dependence only through the transfer function $H$ ). The dependence of the gain $k$ is hyperbolic, the value of the efficiency can reach large magnitudes. An analysis of this scheme shows that the presence of a second group of sensors and an intermediate plate is optional. It is sufficient, so that the compensating force $Q a$ acts on the foundation after the force sensors of the first group. AVS work control in this case may be carried out using the foundation vibration.

\section{CONCLUSION}

Possible schemes for active broadband systems of vibrational forces damping, transferred to the foundation through the vibration isolation of the installation have been analyzed. Consideration of a visual symmetric single-mass system allowed identifying their principal features. Due to the assumed simplifications schemes marginal efficiencies have been identified; their differences and their influence on the physical properties of the original system have been shown. The influence of the unaccounted real factors (such as the compliance of the foundation and force sensors, the dynamic properties of the active vibration damper, the damping values) on the efficiency of various AVS schemes has been studied in sufficient detail in the scientific papers.

The choice of the AVS scheme can significantly affect the dynamic characteristics of a vibration-insulated object and the AVS efficiency. When choosing an AVS scheme with the active force influence on the force sensors under a vibration-insulated object (on the foundation, schemes № 3 and № 5), the amplitude-frequency characteristic of the 
object does not change, and the AVS efficiency does not depend explicitly on the frequency. The implementation of the AVS according to scheme №5 with two groups of force sensors eliminates the feedback loop in the AVS and the vibration-insulated object influence on the dynamic properties that can provide a better AVS efficiency compared with other schemes (Also the see the scientific research paper [1]).

\section{ACKNOWLEDGMENT}

The paper was written on account of the Russian Science Foundation (the RSF) Grant. Agreement No. 16-19-10292 dated May 12, 2016 between the Russian Science Foundation and Kaluga State University named after K.E. Tsiolkovsky.

\section{REFERENCES}

1. M.D. Genkin, V.G. Elezov, V.V. Yablonsky. Methods of Active Vibration Damping, in a collection of articles. Dynamics and Acoustics of Machines. Moscow: Science, pp. 70 - 88, 1971.

2. M.D. Genkin, V.G. Elezov, V.V. Yablonsky. Controlled Vibration Protection Methods for Machines. Moscow: Science, pp. 240, 1985.

3. A.V. Kiryukhin, V.A. Tikhonov, A.G. Chistyakov, V.V. Yablonsky. Active Vibration Protection Function, principles, present state. The function and principles of development. Problems of mechanical engineering and automation, 2, 2011, pp. 108-111.

4. A.V. Kiryukhin, O.O. Milman, A.V. Ptakhin, L.N. Serezhkin. Reduction of Vibration Forces and Pressure Pulsations in the Pipeline Compensators with Passive and Active Methods. International Journal of Mechanical Engineering and Technology, 9(9), 2018, pp. 1452-1462. 